\title{
FATAL CELLULITIS CAUSED BY APOPHYSOMYCES ELEGANS
}

\author{
*AJ Kindo, NR Shams, K Kumar, S Kannan, S Vidya, AR Kumar, J Kalyani
}

\begin{abstract}
A case of cellulitis of the left lateral side of the face caused by the zygomycete Apophysomyces elegans in a healthy male following a road traffic accident is reported. The contaminated soil was the source of fungus. Broad aseptate fungal hyphae were seen in the necrosed tissues. Extensive tissue debridement and treatment with amphotericin B were not successful in controlling the rapid invasion of the tissues by the fungus. Patient developed angioinvasion, severe cellulitis and finally succumbed to the infection three weeks after admission.
\end{abstract}

Key words: Apophysomyces elegans, cellulitis, zygomycetes

Apophysomyces elegans is classified within the family mucorales of the class Zygomycetes. ${ }^{1}$ It is a filamentous fungus occurring in soil and decaying vegetation as a common environmental contaminant. It is commonly found in tropical and subtropical regions. ${ }^{2}$ The species was first isolated in 1979 from soil samples collected in a mango orchard in northern India. ${ }^{3}$ The first human infection caused by this fungus was described in 1985 and since then quite a few cases have been reported in English literature. Most of the reported cases have come from regions with warm climates, such as the southern portions of the United States, Mexico, North Australia, and South India. We report a case for the rarity of the clinical picture.

\section{Case Report}

A 49-year-old male was brought to the emergency room with swelling and redness of the left side of the face extending to the left ear and neck, with signs of perichondritis and facial nerve palsy. He had deep cut injury over the left parietal area, laceration of the scalp, and left external ear due to a road traffic accident six days prior to this. The laceration had been sutured in another hospital.

There was no history of loss of consciousness, vomiting, or altered sensorium. Physical examination revealed the patient to be conscious, well oriented, afebrile, and normotensive. The patient also had bilateral acromioclavicular joint dislocation.

Cardiovascular system and respiratory system examination were normal. The patient was a known diabetic on irregular medication.

\footnotetext{
*Corresponding author (email:<anupmalakra@yahoo.com>) Departments of Microbiology (AJK, NRS, JK), Infectious Disease (KK, SV) and ENT and Head and Neck Surgery (SK, ARK), Sri Ramachandra Medical College and Research Institute, Chennai - 600 116, India
}

Received: 16-11-06

Accepted: 10-02-07
Routine laboratory parameters at the time of admission were as follows: hemogloblin, $97 \mathrm{~g} / \mathrm{L}$; total count, $31.9 \times$ $10^{9} / \mathrm{L}$; random blood sugar, $25.9 \mathrm{mmol} / \mathrm{L}$; and urine positive for ketone bodies and sugar. Other parameters were within normal limits. Chest X-ray was normal. A diagnosis of diabetic ketoacidosis with cellulitis was arrived at.

Radical debridement of the left parietal, facial and neck area was done.

Tissue material was sent for mycological, bacteriological and histopathological examination. The tissue was minced and subjected to potassium hydroxide $(\mathrm{KOH})$ examination. The $\mathrm{KOH}$ mount showed aseptate fungal elements (Fig. 1). After 72 hours of incubation, fungal culture showed white cottony growth with abundant aerial hyphae with no reverse pigment on Sabouraud dextrose agar (Hi-Media).

Lactophenol cotton-blue mount showed numerous sparsely septate ribbon like fungal hyphae with few rhizoids suggestive of zygomycetes but with no sporulation.

Sporulation was induced by water culture and incubated at $37^{\circ} \mathrm{C}$. After seven days, microscopy showed sporangiophores having funnel-shaped apophyses and pyriform sporangia characteristic of A. elegans (Fig. 2).

Histological examination showed broad, sparsely septate, thin-walled hyphae with extensive areas of necrosis, cellulitis, and hemorrhage. No pyogenic organisms were isolated either from the wound or from the blood.

The patient was started on amphotericin B, following which he developed hypersensitive reaction to this drug and injection hydrocortisone had to be given.

There was an aggressive growth of the fungus grossly visible on the periphery of the debrided area. By the twentieth day, the patient's condition deteriorated and he developed signs of angioinvasion. CT scan of the brain showed right cerebral artery territory infarct and liver function tests showed 


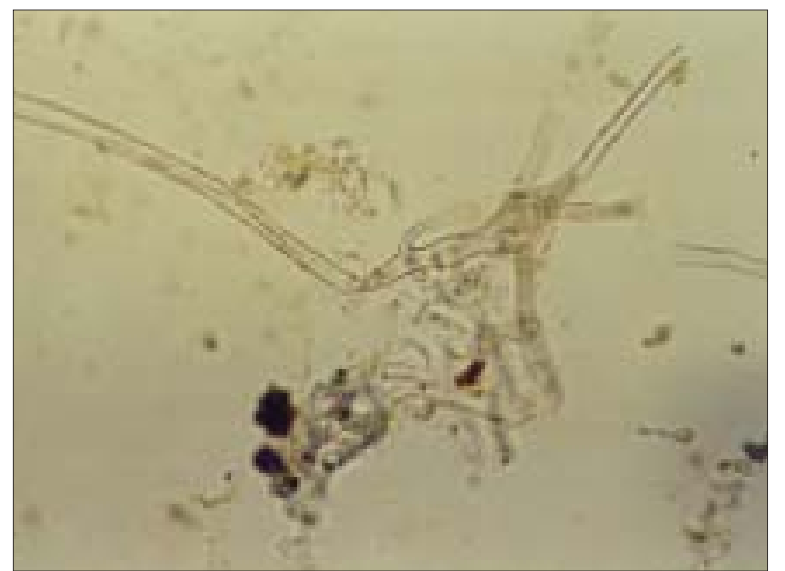

Figure 1: $\mathrm{KOH}$ mount of the tissue showing broad aseptate hyphae $(\times 200)$

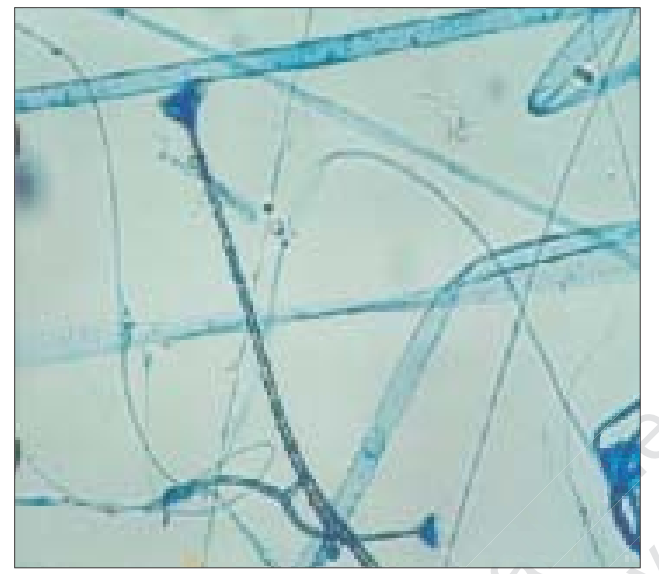

Figure 2: Lactophenol cotton-blue mount of Apophysomyces elegans showing sporangiophores having funnel-shaped apophyses and pyriform sporangia $(\times 200)$

hyperbilirubinemia. There was an increased total count with polymorphs as $90 \%$ and ESR was raised. The patient finally succumbed to the fungal infection.

\section{Discussion}

The zygomycoses are infections caused by fungi of the class Zygomycetes, comprising of the orders Mucorales and Entomophthorales. The Entomophthorales are rare causes of subcutaneous and mucocutaneous infections known as entomophthoromycosis, which largely affect immunocompetent hosts.

Fungi belonging to the order Mucorales are distributed into six families, all of which can cause cutaneous and deep infections. Species belonging to the family Mucoraceae are isolated more frequently from patients with mucormycosis than any other family. Among the Mucoraceae, Rhizopus oryzae (rhizopus arrhizus) is by far the most common cause of infection. ${ }^{4}$

Other less frequently isolated species of the Mucoraceae family that cause a similar spectrum of infections includes
Rhizopus microsporus var rhizopodiformis, Absidia corymbifera, A. elegans, Mucor spp., and Rhizomucor pusillus. $^{4}$

A. elegans is a rare cause of human Zygomycosis ${ }^{4,5}$ and is usually acquired via traumatic implantations, insect bites, surgery and contamination of burn wounds. Invasive soft tissue infections develop on burns or wounds contaminated by soil.

A. elegans infections present most commonly as necrotizing fasciitis, osteomyelitis and angioinvasion..$^{5-7}$ Systemic and secondary renal and bladder infections ${ }^{8,9}$ have also been reported. A. elegans tends to invade and grow within the vascular lumen like other species of the zygomycetes. Vascular invasion frequently causes thrombosis leading to ischaemic tissue necrosis. ${ }^{10}$ Histopathological examination of most infected tissue with $A$. elegans has revealed hyphae in the thrombi. The rapid progress of necrosis, which usually occurs within days after inoculation, may be explained by the rapid growth within the vessels.

A hallmark of mucormycosis infections is the virtually uniform presence of extensive angioinvasion. This angioinvasion is associated with the ability of the organism to hematogenously disseminate from the original site of infection to other target organs. Hence, damage of and penetration through endothelial lining of blood vessels is likely to be a critical step in the pathogenesis of this organism. There was a delay in the antifungal treatment as the patient was brought to the hospital after cellulitis had spread extensively.

In vitro susceptibility data reported so far are very limited. Minimum inhibitory concentration (MIC) breakpoints for interpretation of in vitro susceptibility results have not been defined. Some of the isolates may yield relatively low amphotericin B MICs (in micrograms per milliliter). Amphotericin B appears as the sole antifungal drug, which may be active against $A$. elegans similar to the other members of the class Zygomycetes. Extensive surgical debridement and amphotericin B therapy may be efficacious in vivo. ${ }^{9}$

This case highlights the importance of fungal agents like A. elegans that can cause infection in previously healthy patients who suffer an injury to the cutaneous barrier. Infection with this Zygomycete should be considered when there is progressive necrosis of a wound in a previously healthy individual especially when there is an ineffective response to antibacterial chemotherapy.

\section{Acknowledgement}

We specially thank Dr. Arunaloke Chakrabarti, PGIMER, Chandigarh, for confirming the identification of our isolate.

\section{References}

1. Kwon-Chung KJ, Bennett JE. Mucormycosis. In: Medical Mycology. Pa: Lea and Febiger: Philadelphia; 1992. p. 524-59. 
2. Collier L, Balows A, Sussman M. Topley and Wilson's Microbiology and Microbial infections, $9^{\text {th }}$ ed, vol.4. Arnold: London; 1998.

3. Misra PC, Srivastava KJ, Lata K. Apophysomyces, a new genus of Mucorales. Mycotaxon 1997;8:377-82.

4. Ribes JA, Vanover Sams CL, Baker DJ. Zygomycetes in human disease. Clin Microbiol Rev 2000;13:236-301.

5. Lawrence RM, Snodgrass WT, Reichel GW, Padhye AA, Ajello L, Chandler FW. Systemic Zygomycosis caused by Apophysmyces elegans. J Med Vet Mycol 1986;24:57-65.

6. Cooter RD, Lim IS, Ellis DH, Leitch IO. Burn wound zygomycosis caused by Apophysmyces elegans. J Clin Microbiol 1990;28:2151-3.

7. Lakshmi V, Rani TS, Sharma S, Mohan VS, Sundaram C,
Rao RR, et al. Zygomycotic necrotizing fasciitis caused by Apophysmyces elegans. J Clin Microbiol 1993;31:1368-9.

8. Lawrence RM, Snodgrass WT, Reichel GW, Padhye AA, Ajello L, Chandler FW. Systemic Zygomycosis caused by Apophysmyces elegans. J Med Vet Mycol 1986;24:57-65.

9. Okhuysen PC, Rex JH, Kapusta M, Fife C. Successful treatment of extensive posttraumatic soft-tissue and renal infections due to Apophysmyces elegans. Clin Infect Dis 1994;19:329-31.

10. Kimura M, Smith MB, McGinnis MR. Zygomycosis due to Apophysmyces elegans: Report of 2 cases and review of the literature. Arch Pathol Lab Med 1999;123:386-90.

Source of Support: Nil, Conflict of Interest: None declared. 